\title{
Realization of the Relationship between Quantum Mechanics and Relativity
}

\author{
Mohamed Daris* \\ Department of Physics, University of Sciences, Morocco
}

*Corresponding Author: Mohamed Daris, Department of Physics, University of Sciences, Morocco

\begin{abstract}
Relativity and quantum mechanics remain two very binding things and are both separating either by method or by physical experience. In this section I will make an approach to better facilitate a connection between quantum mechanics and relativity that gives us a new vision on the fact that the two concepts can be related theoretically and experimentally to give birth to the new principles in the physical theory and to better improve theoretical and experimental research. The new conception of this principle develops scientific and technical research better.
\end{abstract}

Keywords: energy volume; energy mass; energy density; matter; quantum mechanics; speed of the light;mass.

\section{AbreViations :}

M:the mass of matter ; v: the volume ; $\rho$ :density ; $\Psi$ :wave function ; Em: enery of mass; E $\rho$ :energy density ; EV:energy volume ; c: speed of light ; TF : final time of the universe.

\section{INTRODUCTION}

In this section we will have the design of a central energy model based on the energy of the light $\mathrm{E}$ this model has three forms of energy: the energy of the mass $E_{m}$ which is based deep on the mass $m$ in relation with the wave function $\Psi$ and the density density energy $E_{\rho}$ which is based on the density density $\rho$ in relation to the wave function $\Psi$ and the volume energy $E_{V}$ which is based on the volume $\mathrm{V}$ in relation with the wave function $\Psi$. This approximation gives us access to the last very important paragraph in this research is the relationship between quantum mechanics and relativity.

\section{DISCUSSION}

\section{Realization of the relationship between quantum mechanics and relativity:}

I) Relationship building for the model $\left(\mathbf{E}_{\mathrm{m}}, \mathbf{E}_{\boldsymbol{\rho}}, \mathbf{E}_{\mathrm{V}}\right)$ :

We have:

$E=\Psi C^{2} \sqrt{T_{F}} \quad ; \quad m=\frac{E}{C^{2} \sqrt{T_{F}}}=\frac{\Psi C^{2} \sqrt{T_{F}}}{\sqrt{\rho}} \times \frac{1}{C^{2} \sqrt{T_{F}}}=\frac{\Psi}{\sqrt{\rho}}$

And we know that: $\quad m=\frac{\Psi}{\sqrt{\rho}}$

So: $\quad \rho=\frac{\Psi^{2}}{m^{2}} \quad ; \quad V=\frac{m}{\rho}=\frac{\Psi}{\sqrt{\rho}}=\frac{m^{2}}{\Psi^{2}}=\frac{m^{2}}{\sqrt{\rho} \Psi}$

$m=\frac{\Psi}{\sqrt{\rho}} \quad ; \quad \rho=\frac{\Psi^{2}}{m^{2}} \quad ; \quad V=\frac{m^{2}}{\sqrt{\rho} \Psi}$

- Model design $\left(\mathrm{E}_{\mathrm{m}}, \mathrm{E}_{\rho}, \mathrm{E}_{\mathrm{V}}\right)$ :

We have:

$$
m=\frac{E}{C^{2} \sqrt{T_{F}}}=\frac{\Psi}{\sqrt{\rho}} \quad ; \quad \rho=\left(\frac{\Psi \mathrm{c}^{2}}{E}\right)^{2} \times T_{F}=\frac{\Psi^{2}}{m^{2}} \quad ; \quad V=\frac{E}{\mathrm{c}^{2} \Psi^{2} \sqrt{T_{F}}}=\frac{m^{2}}{\sqrt{\rho} \Psi}
$$


For $\mathrm{E}_{\mathrm{m}}$ we have:

$\frac{E}{C^{2} \sqrt{T_{F}}}=\frac{\Psi}{\sqrt{\rho}}$

So:

$E=\frac{\Psi \mathrm{c}^{2} \sqrt{T_{F}}}{\sqrt{\rho}}$

$E_{m}=\Psi \mathrm{c}^{2}\left(\frac{T_{F}}{\rho}\right)^{\frac{1}{2}}$

For $\mathrm{E}_{\rho}$ we have:

$\left(\frac{\Psi \mathrm{c}^{2}}{E}\right)^{2} \times T_{F}=\frac{\Psi^{2}}{m^{2}}$

So:

$\frac{\Psi C^{4}}{E^{2}} \times T_{F}=\frac{\Psi^{2}}{m^{2}}$

$E_{\rho}=m c^{2} \sqrt{T_{F}}$

So:

$$
E_{\rho}=\frac{\Psi}{\sqrt{\rho}} c^{2} \sqrt{T_{F}}=\Psi c^{2}\left(\frac{T_{F}}{\rho}\right)^{\frac{1}{2}}
$$

$E_{\rho}=\Psi \mathrm{c}^{2}\left(\frac{T_{F}}{\rho}\right)^{\frac{1}{2}}$

For $\mathrm{E}_{\mathrm{v}}$ we have:

$V=\frac{E}{c^{2} \Psi^{2} \sqrt{T_{F}}}=\frac{m^{2}}{\sqrt{\rho} \Psi}$

So:

$$
E=m^{2} c^{2} \Psi\left(\frac{T_{F}}{\rho}\right)^{\frac{1}{2}}
$$

So:

$$
E_{V}=m^{2} c^{2} \Psi\left(\frac{T_{F}}{\rho}\right)^{\frac{1}{2}}
$$

So the 3 final results are:

$E_{m}=\Psi \mathrm{c}^{2}\left(\frac{T_{F}}{\rho}\right)^{\frac{1}{2}} \quad ; \quad E_{\rho}=\Psi \mathrm{c}^{2}\left(\frac{T_{F}}{\rho}\right)^{\frac{1}{2}} \quad ; \quad E_{V}=m^{2} \mathrm{c}^{2} \Psi\left(\frac{T_{F}}{\rho}\right)^{\frac{1}{2}}$

We can deduce that: $\quad E_{m}=E_{\rho}=\Psi_{\mathrm{c}}^{2}\left(\frac{T_{F}}{\rho}\right)^{\frac{1}{2}}$

So we have:

$$
E_{V}=E_{\rho} m^{2}=E_{m} m^{2}
$$

- these results show that volume-related energy $E_{v}$ is very large compared to the energy related to the mass energy $E_{m}$ or density $E_{\rho}$ which are identical.

We have: $\quad \rho=\frac{\Psi^{2}}{\mathrm{~m}^{2}}$

so: $\quad m^{2}=\frac{\Psi^{2}}{\rho}$

So we will have the new relationship:

$E_{V}=m^{2} c^{2} \Psi\left(\frac{T_{F}}{\rho}\right)^{\frac{1}{2}}=\frac{\Psi^{3} c^{2}}{\rho}\left(\frac{T_{F}}{\rho}\right)^{\frac{1}{2}}$ 
So:

$$
E_{V}=\frac{\Psi^{3} \mathrm{c}^{2} \sqrt{T_{F}}}{(\rho)^{\frac{3}{2}}}
$$

- We have:

$$
E_{V}=E_{\rho} m^{2}=E_{m} m^{2}
$$

so:

$$
E_{V}=E_{\rho} \frac{\Psi^{2}}{\rho}=E_{m} \frac{\Psi^{2}}{\rho}
$$

So we have the stability of the total matter:

$\rho E_{V}=E_{\rho} \Psi^{2}$

And we have:

$$
\rho E_{V}=E_{m} \Psi^{2}
$$

So we have the density of matter is exist in two forms of energy: mass energy and energy density. In a global form of energy, which is the energy density, we have:

$\rho=\frac{E_{\rho} \Psi^{2}}{E_{V}}=\frac{E_{m} \Psi^{2}}{E_{V}}$

- This relation is the total stable energy equilibrium relation of matter or of a body; system; universe ... that has an energy $E_{m} ; E_{\rho} ; E_{V}$. This relationship represents a link between quantum mechanics and relativity.

\section{The final equation of the connection between relativity and quantum mechanics is:}

We pose:

$E_{\rho}=E_{m}=E$

So we will have:

$\rho=\frac{E \Psi^{2}}{E_{V}}$

So the final equation is this:

$E=\frac{\rho E_{V}}{\Psi^{2}}$

-This equation Is the Link between relativity and quantum mechanics shows that energy

$E=m c^{2} \sqrt{T_{F}}$ Is linked with the wave function $\Psi$ according to density $\rho$ in a volumic energy present $\mathrm{E}_{\mathrm{V}}$. This result is final for this link.

- Physical interpretation of the results exploited:

Energy $E=m c^{2} \sqrt{T_{F}}$ is an exact correction of the classical light equation: $E=\mathrm{mc}^{2}$; the first equation shows that the photon, the light, the geo matter as the planets the galaxies, the stars the black holes all depend on the spatial time parameter $T_{F}$;

and consequently their energy produced depends on the matter of the mass and the speed of the light produced at the level of this body or this system means that the wave function $\Psi$ is

depends on quantum mechanics and e relativity at the same time it's an essential parameter in the equation:

$$
E=\frac{\rho E_{V}}{\Psi^{2}} .
$$

The density $\rho$ and energy $E_{V}$ represents the complex state of matter that has energy density $E_{V}$ and a density $\rho$.

This equation is a proper state of the presence of relativity and quantum mechanics at the same time in any system on the universe or far from the universe. 


\section{REFERENCES}

[1] Ferrarese, Laura; Merritt, David (2000). "A fundamental relation between supermassive black holes and their host galaxies". AstrophysicalJournalLetters. 539 (1):L9L12. arXiv:astroph/0006053 . Bibcode:2000ApJ...539L... 9F. doi:10.1086/312838.

[2] S. Lloyd, «Ultimate physical limits of computation », Nature, vol. 406, no 6799, 2000, p. 10471054 (PMID 10984064,DOI 10.1038/35023282, Bibcode 2000Natur.406.1047L, arXiv quant-ph/9908043)

[3] Laura Merritt et David Merritt, «A fundamental relation between supermassive black holes and their host galaxies », Astrophysical Journal Letters, vol. 539, no 1, 2000, p. L9L12 (DOI 10.1086/312838, Bibcode 2000ApJ...539L...9F, arXiv astro-ph/0006053)

[4] The relative permeability and permittivity of field-theoretic vacuums is described in Kurt Gottfried; Victor Frederick Weisskopf (1986). Concepts of particle physics.

[5] Oxford University Press. p. 389. ISBN 0-19-503393-0. and more recently in John F. Donoghue; Eugene Golowich; Barry R. Holstein (1994). Dynamics of the standard model. Cambridge University Press. p. 47. ISBN 0-521-47652-6. and also R. Keith Ellis;

[6] W. J. Stirling; B. R. Webber (2003). QCD and collider physics. Cambridge University Press. pp. 2729. ISBN 0-521-54589-7. Returning to the vacuum of a relativistic field theory, we find that both paramagnetic and diamagnetic contributions are present. QCD vacuum is paramagnetic, while QED vacuum is diamagnetic. See Carlos A. Bertulani (2007). Nuclear physics in a nutshell. Princeton University Press. p. 26. ISBN 0-691-12505-8.

Citation: Mohamed Daris, Realization of the Relationship between Quantum Mechanics and Relativity, International Journal of Scientific and Innovative Mathematical Research (IJSIMR), vol. 8, no. 7, pp. 14-17, 2020. Available : DOI: https://doi.org/10.20431/2347-3142.0807004

Copyright: (C) 2020 Authors. This is an open-access article distributed under the terms of the Creative Commons Attribution License, which permits unrestricted use, distribution, and reproduction in any medium, provided the original author and source are credited. 